\title{
Interdisciplinary Design of a Pervasive Fall Handling System
}

\author{
Jan Van den Bergh, Kris Luyten \\ Hasselt University - tUL - iMinds \\ Expertise Centre for Digital Media \\ Wetenschapspark 2, Diepenbeek, Belgium \\ firstname.lastname@uhasselt.be \\ Brenda Aendekerk \\ Wit-Gele Kruis Limburg VZW \\ Welzijnscampus 25 \\ 3600 Genk, Belgium \\ brenda.aendekerk@limburg.wgk.be
}

A case study

\author{
Shirley Elprama, An Jacobs \\ VUB - SMIT - iMinds \\ Pleinlaan 9 \\ 1050 Brussels, Belgium \\ firstname.lastname@vub.ac.be \\ Femke De Backere \\ Department of Information Technology (INTEC) \\ Ghent University - iMinds, Gaston Crommenlaan \\ 8 bus 201 \\ B-9050 Ghent, Belgium \\ femke.debackere@intec.ugent.be
}

\begin{abstract}
Falls among elderly are an important concern as they impact the capability to live independently. Falls do not only have a negative impact on one's physical well-being, an increased risk of falling also has an important impact on one's psychological well-being. A context-aware fall handling system can mitigate many of the problems of falls by facilitating timely and appropriate handling of falls.

In this paper, we present the results of an early exploration of using context as part of fall detection and resolution services in Flanders, Belgium. We put specific emphasis on the role of diverse disciplines during the process and reflect on their influence on the outcomes. We believe that these reflections could be more generally applicable for interdisciplinary research projects.
\end{abstract}

\section{Categories and Subject Descriptors}

D.2.1 [Requirements/Specifications]: Elicitation methods (e.g., rapid prototyping, interviews, JAD); H.5.2 [User Interfaces]: User-centered design

\section{General Terms}

Design, Human Factors

\section{Keywords}

Context awareness, fall handling, interaction design
Permission to make digital or hard copies of all or part of this work for personal or classroom use is granted without fee provided that copies are not made or distributed for profit or commercial advantage and that copies bear this notice and the full citation on the first page. To copy otherwise, to republish, to post on servers or to redistribute to lists, requires prior specific permission and/or a fee. IDPHA 2014, May 20-22

Copyright (C) 2014 ICST 978-1-63190-011-2

DOI 10.4108/icst.pervasivehealth.2014.255784

\section{INTRODUCTION}

Falls among elderly are a problem, as they can have both physical as psychological consequences. Elderly can ask for help after a fall by using a Personal Emergency Response System (PERS). This is small device consisting of a button and a receiver. Elderly can wear this button around their neck or wrist and press it in case of an emergency. The receiver will send the signal to a call center and opens a 2-way speech/listen connection between the person in emergency and a second party, in our case a qualified care organization. The performance of this system, though already very useful, is far from perfect and there are several ways to improve upon it. One way is to integrate automated sensors, e.g. camera-based fall detection [3], microphone arrays [5] or wearable sensors [1], another way is working on the operating procedures when a fall is detected. This paper focuses on the fall handling procedure during which patients will be provided with appropriate help through a call center in the province of Limburg, Belgium. Currently, a desk operator, which in this case is always a trained, practicing nurse, will be put in contact with the patient and, in case this is needed, will call contacts (such as family members and neighbors) in a given order, until one is able to go to the patient's home.

One way to save time is to skip the entire call center and immediately inform all contacts with a picture of the situation and the option to start a live video stream as proposed by the incubating start-up FamilyEye ${ }^{1}$. This has the limitation that a relatively close social network (both in terms of relationship and space) is required. Furthermore, it is difficult for contacts to judge the situation when they lack in-depth medical knowledge. The Florence project ${ }^{2}$ takes another approach by letting a robot do a first diagnosis and provide information to a call center. They did not investigate further call handling.

We propose to keep the call center as part of the process and

${ }^{1}$ http://www. familyeye.be/

${ }^{2}$ http://www.florence-project.eu 


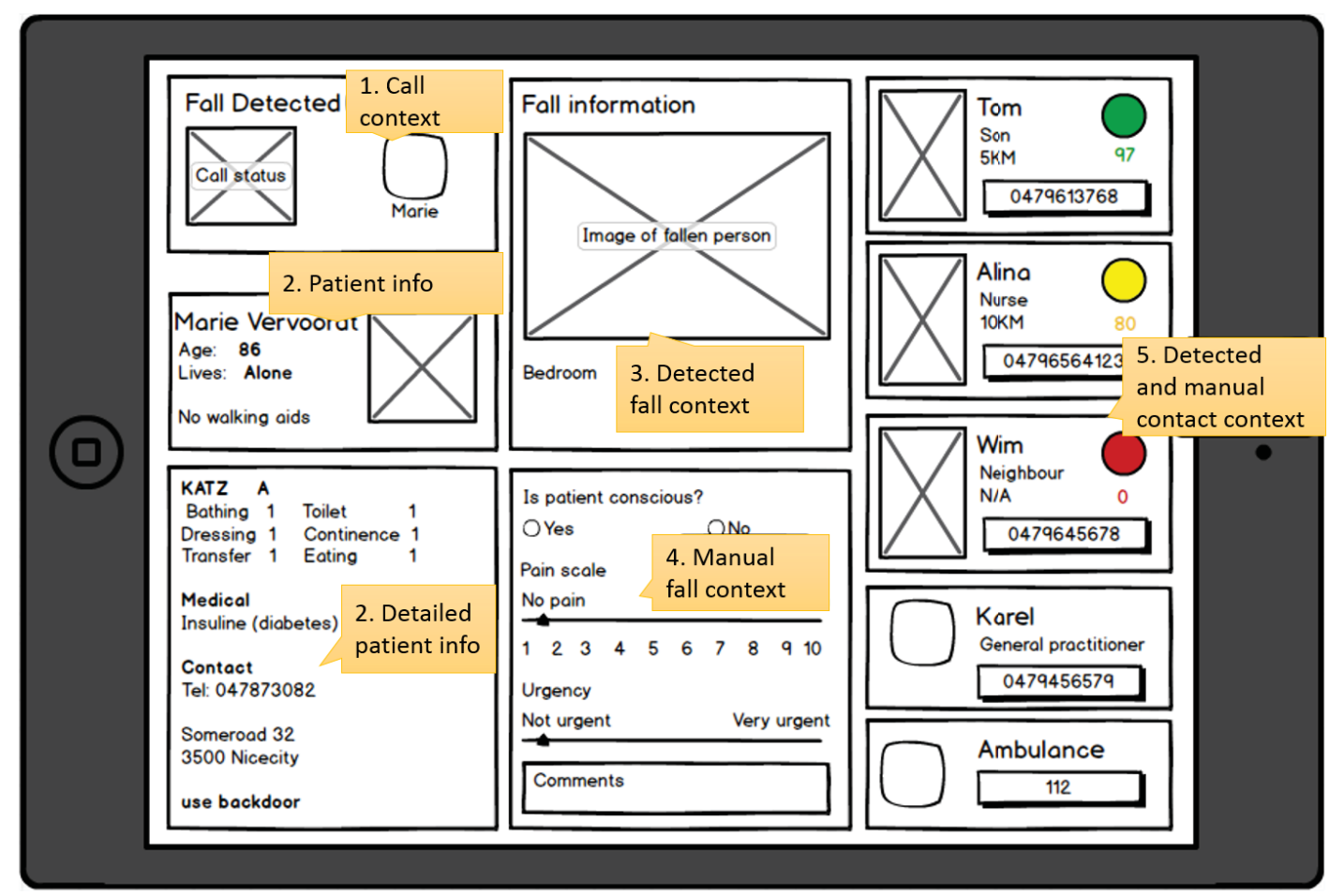

Figure 1: Structure of the design used during the last interviews. Subsequent iterations information had additional information added all parts except part 1. Note: The actual designs had a more finished look.

in control of the fall handling procedure, but we propose to use a mixed initiative approach [6] in which the interactive computing system, desk operator and (informal) caregivers cooperate to select the most appropriate contact. This approach exploits certain dynamic (context) information about (informal) caregivers, gathered using a smartphone, such as location or availability (based on calendar or ringer profile) without interaction from the user. Given this richer information, one can let the system automatically order the contacts and increase the chance of quickly contacting a person that will attend the patient.

This paper discusses our findings on working procedures and user interface for the desk operator to take advantage of context-aware information available through current technology using such a mixed-initiative approach. Interviews with nurses and other employees of a home care organization provided input for the redesign, by presenting and discussing prototypes of the interface with them. We conclude the paper by some lessons learned both regarding fall handling and the user-centered inter-disciplinary approach used to design the fall handling procedure and interface.

\section{APPROACH}

\subsection{Methodology}

To learn more about the context in which the desk operators work, we did a contextual interview in the call center of a home care organization. This organization provides PERS as a service to their clients. At the call center, a desk operator showed us the alarm and the receiver, explained us in detail how PERS works and what procedure the desk operators follow once they have a call. We observed several times how they handle false alarms as they received a couple false alarms during our observation.

Based on the initial view on the current workflow, humancomputer interaction researchers created a first interactive prototype using Indigo Studio, a software package to create prototypes and wireframes. This prototype was first shown to a researcher of the home care organization. During this 'dry run' a domain expert from the home care organization tested whether the prototype was clear. Based on her feedback, we made some changes to the prototype, such as adding the phone number (lower left in Figure 1) about the patient.

We had three sessions with in total eight interviews (2 male; 6 female) to do formative evaluations of the designed user interface (Figure 1). The interviewees were all working at the home care organization and they had the following roles: six were home care nurses and two were support staff. The latter perform non-medical tasks to alleviate the work load of the nurses. Both participating members of the support staff install the PERS at patient homes, one also attends calls when there is uncertainty about the need for medical follow-up. All interviewed nurses had a specialization: two worked as a desk operator and four were specialized in fall prevention. The age of all interviewees ranged from 22 to 62 years old $($ mean $=39.6$ years; s.d. $=15.4$ years $)$.

After each of the three sessions, we used the gathered feedback to improve the prototype, which was used during the next session. During each interview, we briefly explained the context of the project followed by an introduction of the 
redesigned interface on a tablet. We asked each interviewee to explain what they saw on the screen and to give their opinion about it. After discussing the general interface, we walked the interviewee through each element of the prototype in more detail and asked them for feedback. Example questions include: "Does this part of the user interface provide value?", "Is there information missing?", "When do you need this information?"). Finally, we asked them to give an overall impression of the prototype. During each interview, notes were taken and each interview was recorded on audio. Screenshots of the prototype's interface were printed on paper to quickly note comments related to specific parts of the interface. As the changes made in between the sessions got positive feedback, we did not show older versions of the prototype to the interviewees.

\subsection{Research team}

The approach outlined in the previous section was executed by a research team spread over several research groups and organizations with expertise from different disciplines: socioeconomic impact of new technologies (SOC), human-computer interaction (HCI), engineering (ENG) related to (a.o.) fall detection and smart reasoning systems. The work reported on in this paper was primarily carried out by HCI and SOC researchers. The contextual interviews were led by a SOC researcher with contributions from HCI researchers, while the HCI researchers led the interviews and prototype creation. The other disciplines were invited to give feedback on the created prototypes and were informed about the results of the interviews, including comments on the prototypes.

\section{RESULTS}

\subsection{Current approach}

The initial observations in the call center and the information received at this stage, informed us about the overall workflow of handling a call, which sufficed to get a good initial understanding of the call handling protocol. The current protocol is to have a list of five ${ }^{3}$ contacts (such as family members or neighbors) that will be contacted once a patient needs help. These contacts indicated in which order they want to be contacted in case of an emergency. Unless the patient especially requests a specific person to come or in case contacts indicated they would be on holiday, which is frequently forgotten, this order is respected. However, this method has some disadvantages as contacts are not always available or are too far away to quickly reach the patient after a fall.

Once a patient presses the alarm button, the details such as contact information of this patient are shown on the computer of the desk operator at the call center. Information that is shown includes the list of contacts but also specific information like 'has diabetes' or 'does not hear well'.

Interviewees indicated priorities and selection rules that apply in handling in fall;

1. Is there a fall? There are many (intentional) false positives using the PERS system as patients are expected

${ }^{3}$ This was the ideal number for this particular alarm central, other centrals might prefer up to 10 persons. Patients may have less contacts. to regularly check correct operation of the system and they sometimes accidentally press the alarm button.

2. Can all contacts enter the patient's home? If not, the first priority is to find someone with a key to the patients home, starting with the preference of the patient if expressed or following the order established by the caregivers.

3. Who to contact? If the patient's home is accessible, two other questions need to be answered that need more information than available in the PERS: what is the severity of the fall and who can attend the patient? If no unique answer is available, the desk operator goes through the list of (available) caregivers and the first one who answers, who is also available and not too far from the patient, is the one that goes to attend the patient.

4. What was the result of the intervention? The results of all handled calls are logged for future reference.

The fall handling procedure ends when a caregiver at the patient's home reports the results to the desk operator.

\subsection{Context-awareness}

The interviews delivered several useful insights regarding context-awareness. We can divide the context information in three categories: fall context (Can the patient respond? Where did the fall occur? Is there resulting pain? Is the patient mobile?), contact context (Which contacts are available and how quickly can they get access to the patient's home?), and call context (What was the reason for the call and what actions have already been taken?).

\subsubsection{Fall context}

Part 3 of Figure 1 was used to trigger reactions regarding context information related to the fall.

Showing the picture of the user lying down on the ground after the fall can give the desk operator a better idea about the urgency of the incident and possibly injuries from the patient. Yet interviewees mentioned that the patient should give consent to show the image unless no contact could be established and patient safety could be in danger. Another concern mentioned was that the desk operator should not make the diagnosis. Having such concrete information could thus also distract from the actual task which places this responsibility to a person on site.

Some interviewees indicated that showing something like a stick figure instead of the actual image / video could be an alternative that was considered less of an intrusion regarding privacy but could reveal similarly useful information. An indication of the place where the patient fell was considered at least as important as it would allow the contact coming to help to more quickly locate the patient.

\subsubsection{Contact context}

Contextual information about contacts (Figure 1, right: colorcoded status and distance) was greatly appreciated, although some had doubts how many would be willing or would forget to provide certain information (e.g. regarding availability). 
We repeatedly heard remarks related to the lack of updates on longer absences by contacts.

We also heard several stories related to repeated phone calls to contacts that were in the end not reachable or could not attend the fall. This results in several minutes of time lost, especially since contacts can have multiple registered phone numbers such as fixed line and mobile phone(s).

Having automated updates regarding availability and location were thus greatly appreciated. Interviewees were sceptical regarding manual updates to the status of contacts.

\subsubsection{Call context}

In the current fall handling procedures time-outs and regular phone calls are really important to ensure proper handling of a fall. Interviewees would welcome more technological assistance including (semi-)automated notifications when someone arrives at the site or when the fall is handled. For example, knowing that someone is stuck in a traffic jam could reduce the number of potentially inconvenient phone calls (e.g. calls while contact is driving).

\section{DISCUSSION}

This paper explored the benefits that context-awareness could bring to a fall handling system through a user-centered approach, which used a focused interactive user interface prototype and (primarily) semi-structured interviews. The quick iterations proved useful as consecutive interviews could confirm information from earlier interview and in some cases build upon it. The involvement of several disciplines in the process (section 2.2) was valuable because they had largely complementary but also partially overlapping skills and points of view for carrying out the research. Furthermore they were open towards the needs of others.

We believe it was beneficial that the interviewees had different roles in fall handling as it lead to richer insights due to the complementary experiences with the system. While only a minority was desk operator, most could contribute new insights and/or confirm those provided by others. Interviewees that had more experience in attending falls, could generally provide useful information based on this. For example, several testimonials detailed the need of key access to the house of an elder.

The research disciplines (section 2.2) involved in the design of the context-aware system worked in parallel on both the technical and user side of the project. This posed problems despite regular meetings, a shared vision document and workshops involving the diverse disciplines. Several reasons could be identified, which can be more prevalent when working in interdisciplinary teams; working rhythms and communications may not always be sufficiently adapted or specified.

For example, a realistic estimate of what sensor-derived information could be represented in the user interface was not easy to identify partly because this research is carried out in parallel and there were still uncertainties regarding the results that could be obtained.

Regarding communication it is difficult to abide to the three laws of professional communication [4] as the required infor- mation and the way it should be presented in an interdisciplinary team can be highly different from what is needed or expected when communication within one's own discipline.

Context-awareness was strongly appreciated because it could provide the desk operator, contacts and nurses with relevant context information. The interviewees were convinced that adding awareness of the location and reachability of (informal) caregivers could reduce the time it takes to handle a fall. Interviewees were more critical towards the automated inclusion of images or video about the fall, mainly due to privacy and related acceptance concerns.

Future work within the project will focus on further investigation on the opinions of non-professional stakeholders (contacts and patients) on the proposed approach, especially regarding the focused gathering of the contextual data, such as availability and location in the context of the fall handling procedure. The resulting approach could be integrated in an overall platform [2] to combine the context-aware fall handling mechanism proposed in this paper with enhanced fall detection approaches that deliver fall context information.

\section{ACKNOWLEDGMENTS}

The iMinds FallRisk project is co-funded by iMinds (Interdisciplinary Institute for Technology), a research institute founded by the Flemish Government. Companies and organizations involved in the project are COMmeto, Televic Healthcare, TP Vision, Verhaert and Wit-Gele Kruis Limburg, with project support of IWT.

\section{REFERENCES}

[1] G.-C. Chen, C.-N. Huang, C.-Y. Chiang, C.-J. Hsieh, and C.-T. Chan. A reliable fall detection system based on wearable sensor and signal magnitude area for elderly residents. In Aging Friendly Technology for Health and Independence, pages 267-270. Springer, 2010.

[2] F. De Backere, F. Ongenae, F. Van den Abeele, J. Hoebeke, S. Verstichel, A. Ackaert, and F. De Turck. Social-aware and context-aware multi-sensor fall detection platform. In SWAT4LS, 2013.

[3] G. Debard, P. Karsmakers, M. Deschodt, E. Vlaeyen, J. Van den Bergh, E. Dejaeger, K. Milisen,

T. Goedemé, T. Tuytelaars, and B. Vanrumste. Camera based fall detection using multiple features validated with real life video. In Workshop Proceedings of the 7th International Conference on Intelligent Environments, volume 10, pages 441-450, 2011.

[4] J.-l. Doumont. The three laws of professional communication. Professional Communication, IEEE Transactions on, 45(4):291-296, 2002.

[5] Y. Li, K. Ho, and M. Popescu. A microphone array system for automatic fall detection. Biomedical Engineering, IEEE Transactions on, 59(5):1291-1301, 2012.

[6] N. Mahmud, K. Luyten, and K. Coninx. Mixed-initiative context filtering and group selection for improving ubiquitous help systems. In Ambient Intelligence-Software and Applications, pages 155-162. Springer, 2012. 